\title{
Sodium lidar measurements of waves and instabilities near the mesopause during the DELTA rocket campaign
}

\author{
B. P. Williams ${ }^{1}$, D. C. Fritts ${ }^{1}$, J. D. Vance ${ }^{2}$, C.-Y. She ${ }^{2}$, T. Abe ${ }^{3}$, and E. Thrane ${ }^{4}$ \\ ${ }^{1}$ NorthWest Research Associates, Colorado Research Associates division, Boulder, Colorado, USA \\ ${ }^{2}$ Department of Physics, Colorado State University, Fort Collins, Colorado, USA \\ ${ }^{3}$ ISAS/JAXA, Kanagawa, Japan \\ ${ }^{4}$ University of Oslo, Oslo, Norway
}

(Received October 2, 2005; Revised July 24, 2006; Accepted July 31, 2006; Online published September 29, 2006)

\begin{abstract}
The sounding rocket for the DELTA (Dynamics and Energetics of the Lower Thermosphere in Aurora) campaign was successfully launched from Andoya at 00:33 UT on Dec 13,2004. Though it was cloudy at the time of launch, the Weber Na lidar was operating intermittently between 20:00 UT and 23:30 UT on Dec 12, observing $\mathrm{Na}$ density, temperature and meridional wind between 80 and $100 \mathrm{~km}$. Throughout the lidar observations, we observed significant small $\left(\lambda_{z}<5 \mathrm{~km}\right)$ and medium-scale $\left(\lambda_{z} \approx 8-15 \mathrm{~km}\right)$ wave activity producing significant wind and temperature shears. There were unusually large (up to $10 \mathrm{~m} / \mathrm{s}$ and $10 \mathrm{~K}$ amplitudes) perturbations of the vertical wind and temperature profiles at $21 \mathrm{UT}$ with a $3 \mathrm{~km}$ vertical wavelength that was much stronger in the vertical beam than in the north beam. The atmosphere appeared to become more active as the launch time approached. During the last interval with data, at 23:20 UT, Dec. 12th, the lidar profiles revealed a gravity wave in both beams with a magnitude of 5-10 K in temperature and approximately $5 \mathrm{~km}$ vertical wavelength. The large background shear plus the wave perturbation produced regions with potential convective instability at multiple altitudes.
\end{abstract}

Key words: Mesopause dynamics, temperature structure, instabilities, gravity waves.

\section{Introduction}

The sounding rocket for the DELTA (Dynamics and Energetics of the Lower Thermosphere in Aurora) campaign was successfully launched from Andoya Rocket Range at 00:33 UT on Dec 13, 2004. The objective of the campaign was to investigate the response of lower thermospheric dynamics and energetics to auroral energy input, so the rocket was launched into an auroral arc. Imager observations suggest that the arc was located at roughly $120 \mathrm{~km}$ altitude. The nearby Arctic Lidar Observatory for Middle Atmosphere Research (ALOMAR) provided ground-based support for the rocket campaign with instruments such as the Weber sodium lidar, the Rayleigh-Mie-Raman (RMR) lidar, and a meteor and two medium-frequency (MF) radars. The rocket campaign also brought several Fabry-Perot interferometers to the area for additional thermospheric wind diagnostics. Other papers in the DELTA special issue detail the rocket measurements of lower thermosphere temperature (Kurihara et al., 2005) and radar measurements of horizontal wind and electron density (Nozawa et al., 2005), so we will concentrate on the sodium lidar measurements of mesopause-region temperature and winds and their gravity wave perturbations.

The Weber sodium wind-temperature lidar was installed at ALOMAR in 2000 (She et al., 2002) and has pre-

Copyright (c) The Society of Geomagnetism and Earth, Planetary and Space Sciences (SGEPSS); The Seismological Society of Japan; The Volcanological Society of Japan; The Geodetic Society of Japan; The Japanese Society for Planetary Sciences; TERRAPUB. viously supported a number of rocket campaigns, such as MaCWAVE/MIDAS in summer 2002 (Goldberg et al., 2004), winter MaCWAVE in Jan 2003 (Goldberg et al., 2006), DELTA in Dec 2004, and ROMA in Jan 2005. In the summer MaCWAVE/MIDAS campaign, we observed very large positive and negative temperature gradients (Fritts et al., 2004) and large gravity wave variability (Williams et al., 2004). During the winter MaCWAVE campaign, a very large semidiurnal tide caused a large temperature inversion and areas of instability that propagated down with the tidal phase (Williams et al., 2006). The formation of temperature inversions, the creation and evolution of instabilities, and their relationship with atmospheric gravity waves are not fully understood yet and continue to be an active area of research (Li et al., 2005; Hecht et al., 2004; She et al., 2004) with several recent reviews (Meriwether and Gerrard, 2004; Hecht, 2004; Fritts and Alexander, 2003).

During the DELTA campaign, the Weber lidar observed several interesting dynamical features:

1) Significant small $\left(\lambda_{z}<5 \mathrm{~km}\right)$ and medium-scale $\left(\lambda_{z} \approx 8-15 \mathrm{~km}\right)$ wave activity throughout the night.

2) An unusually large perturbation of the vertical wind (up to $10 \mathrm{~m} / \mathrm{s}$ amplitude) and temperature profiles (up to $10 \mathrm{~K}$ amplitude) at 21 UT with a $3 \mathrm{~km}$ vertical wavelength that was much stronger in the vertical beam than in the north beam.

3) A large negative temperature gradient that moved downward with time and enlarged in altitude extent at 22 UT to form a temperature maximum at $84 \mathrm{~km}$ 
Table 1 .

\begin{tabular}{cccll}
\hline $\begin{array}{c}\text { Start time } \\
\text { Hour UT }\end{array}$ & $\begin{array}{c}\text { End time } \\
\text { Hour UT }\end{array}$ & $\begin{array}{c}\text { Number } \\
\text { of files }\end{array}$ & $\begin{array}{l}\text { Random error of } T_{1}, T_{2}, V, W \\
\text { (average from } 82-100 \mathrm{~km} \text { ) }\end{array}$ & $\begin{array}{l}\text { RMS of } T_{1}^{\prime}, T_{2}^{\prime} ; V^{\prime}, W^{\prime} \\
\text { fluctuations }(82-100 \mathrm{~km})\end{array}$ \\
\hline $20: 00$ & $20: 30$ & 24 & $1.3 \mathrm{~K}, 2.0 \mathrm{~K}, 3.0 \mathrm{~m} / \mathrm{s}, 1.5 \mathrm{~m} / \mathrm{s}$ & $10.0 \mathrm{~K}, 12.0 \mathrm{~K} ; 17.0 \mathrm{~m} / \mathrm{s}, 2.5 \mathrm{~m} / \mathrm{s}$ \\
$20: 30$ & $21: 00$ & 29 & $1.0 \mathrm{~K}, 1.3 \mathrm{~K}, 3.0 \mathrm{~m} / \mathrm{s}, 1.0 \mathrm{~m} / \mathrm{s}$ & $8.0 \mathrm{~K}, 8.0 \mathrm{~K} ; 10.0 \mathrm{~m} / \mathrm{s}, 1.5 \mathrm{~m} / \mathrm{s}$ \\
$21: 00$ & $21: 12$ & 12 & $2.4 \mathrm{~K}, 3.0 \mathrm{~K}, 4.5 \mathrm{~m} / \mathrm{s}, 2.0 \mathrm{~m} / \mathrm{s}$ & $16.0 \mathrm{~K}, 16.0 \mathrm{~K} ; 18.0 \mathrm{~m} / \mathrm{s}, 3.0 \mathrm{~m} / \mathrm{s}$ \\
$21: 48$ & $22: 06$ & 15 & $1.8 \mathrm{~K}, 2.2 \mathrm{~K}, 4.0 \mathrm{~m} / \mathrm{s}, 1.5 \mathrm{~m} / \mathrm{s}$ & $6.0 \mathrm{~K}, 7.0 \mathrm{~K} ; 10.0 \mathrm{~m} / \mathrm{s}, 2.5 \mathrm{~m} / \mathrm{s}$ \\
$23: 00$ & $23: 30$ & 19 & $3.6 \mathrm{~K}, 4.0 \mathrm{~K}, 7.0 \mathrm{~m} / \mathrm{s}, 3.0 \mathrm{~m} / \mathrm{s}$ & $9.0 \mathrm{~K}, 9.0 \mathrm{~K} ; 10.0 \mathrm{~m} / \mathrm{s}, 3.5 \mathrm{~m} / \mathrm{s}$ \\
\hline
\end{tabular}

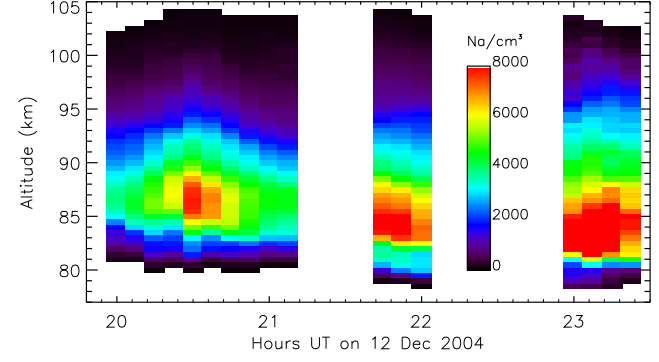

Fig. 1. The sodium density is plotted for beam 1 at $0.5 \mathrm{~km}$ and $7.5 \mathrm{~min}$ resolution to show the time and altitude extent of the data. The data gaps are due to clouds.

with an inversion layer between 82 and $84 \mathrm{~km}$. The large negative temperature gradient was perturbed by a short vertical wavelength gravity wave to form multiple superadiabatic regions that appear to have been convectively unstable.

We begin by describing the Weber lidar and the observations. We will discuss the implications of the three main dynamical features and then compare with previous observations in the last section.

\section{Instrument}

The Weber lidar measures temperature and radial wind by transmitting two $0.5 \mathrm{~W}$ beams with a wavelength of $589 \mathrm{~nm}$ that resonantly scatter from the atmospheric sodium layer located at roughly $80-105 \mathrm{~km}$ altitude. The backscattered light is collected by two $1.8 \mathrm{~m}$ steerable telescopes that are designed to simultaneously and independently receive the signals of the sodium and RMR lidars. We modulate the frequency of the transmitted beams by $\pm 630 \mathrm{MHz}$ every second and third pulse with a pulse repetition rate of $50 \mathrm{~Hz}$ to measure the Doppler shift and broadening of the atmospheric sodium spectrum and determine temperature, radial wind, and sodium density. Beam 1 was oriented at $20^{\circ}$ north of zenith and beam 2 was pointed at the zenith for this campaign, allowing us to measure meridional and vertical wind and temperature profiles at 2 locations separated by $31 \mathrm{~km}$ horizontally at $90 \mathrm{~km}$ altitude. The laser frequency drifted with time due to problems with the frequency locking during this campaign, resulting in a variable drift of the apparent profile-mean vertical wind of 10 to 25 $\mathrm{m} / \mathrm{s}$. This zero-point offset was subtracted from the radial wind measured by both beams. To calculate the meridional wind, we make the usual assumption that the mean vertical wind was zero. But there were also significant perturbations in the vertical wind at short vertical scales, presumably from atmospheric waves, so the small-scale meridional perturbations should be viewed with some caution.

\section{Observations}

Though it was cloudy at the time of launch, the Weber $\mathrm{Na}$ lidar was operating intermittently between 20:00 UT and 23:30 UT on 12 Dec 2004 (day of year 347), observing $\mathrm{Na}$ density, temperature and meridional wind between 80 and $100 \mathrm{~km}$. The observation condition was better between 20:00 UT and 21:12 UT, with near continuous observation. After this time, there were 2 periods of $\sim 15 \mathrm{~min}$ observation, near 22:00 UT and 23:20 UT, respectively. The DELTA rocket was launched one hour later at 00:33 UT on 13 Dec 2004.

Due to the gaps in the data, we divided the lidar data into 5 segments: $20: 00$ to $20: 30$ UT, $20: 30$ to $21: 00$ UT, $21: 00$ to 21:12 UT, $21: 51$ to $22: 06$ UT, 23:00 to 23:06 UT. The data intervals and error levels are summarized in Table 1 . The sodium density is shown in contour format in Fig. 1 for the north lidar beam at $0.5 \mathrm{~km}$ and $7.5 \mathrm{~min}$ resolution to show the time and altitude extent of the data.

The wind and temperature profiles for each data period are shown in Fig. 2. The vertical wind shows little mean gradient with altitude, as expected, but with some significant small vertical scale perturbations. The meridional wind was dominated by a medium scale $(8-12 \mathrm{~km})$ perturbation that moves downward in the first 4 profiles. The two temperature profiles show very good agreement for the large and medium scale features with expected differences at smaller scales due to the $31 \mathrm{~km}$ horizontal separation.

At the beginning of the night, there was a small region with a near-adiabatic lapse rate from 89-92 km which appeared to widen and moved down slightly to $88-92 \mathrm{~km}$ at 20:30-21:00 UT and 87-91 km at 21:00-21:12 UT. After a data gap due to clouds, an apparent temperature maximum was present at $84 \mathrm{~km}$ with near-adiabatic lapse rates from 84 to $93 \mathrm{~km}$ and a large positive temperature gradient below $84 \mathrm{~km}$. After another cloudy period, the temperature maximum moved down slightly to $82-83 \mathrm{~km}$. The wave activity appears to have been stronger, but the noise level also increased due to the clouds that end the lidar observations before the launch of the DELTA rocket.

We calculated the mean temperature and wind profiles by first averaging the first three data periods to even out the time spacing and then averaging this with the last two profiles. The mean temperature profile is shown in Fig. 2 along with MSIS temperature profile for the time and date of the rocket launch, for reference. The MSIS profile is reasonably 

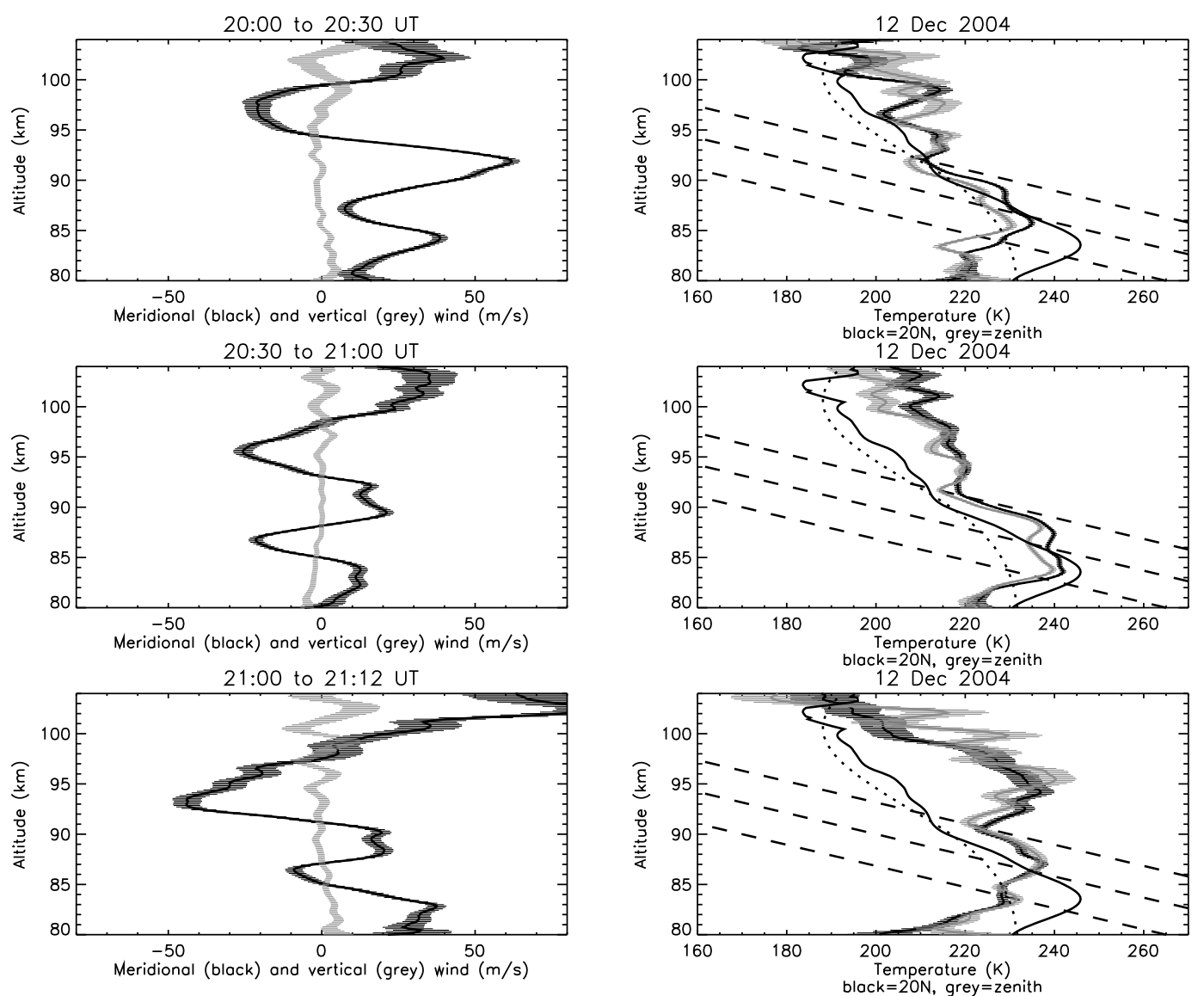

$21: 48$ to $21: 06$ UT
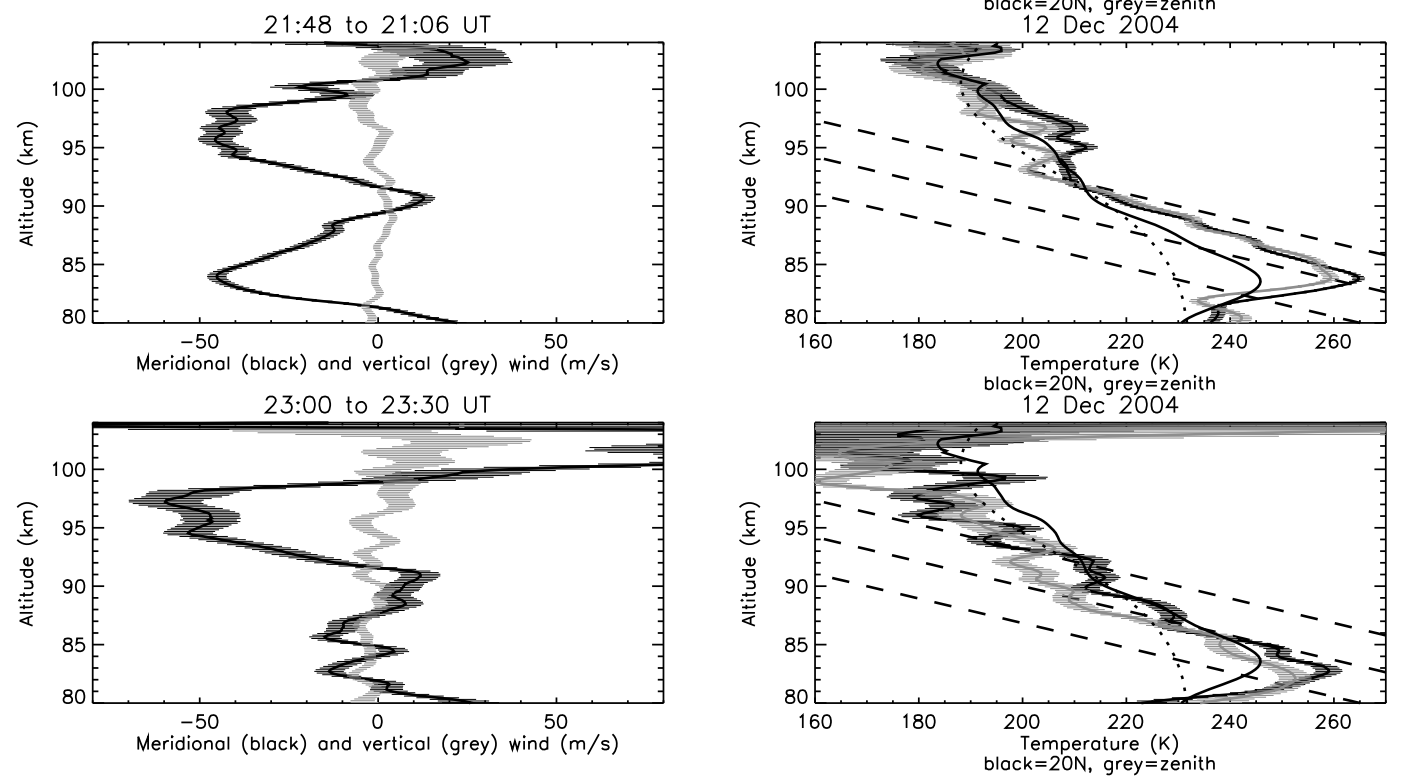

Fig. 2. Left side: For the five time intervals, profiles of meridional wind (positive northward) are shown by black lines and the vertical wind (positive upward) are shown by the grey line with the width giving the random error. Right side: The plots show profiles of temperature for zenith (grey) and north (black) lidar beams for the same five time intervals. Dashed lines indicate the adiabatic lapse rate $(-9.5 \mathrm{~K} / \mathrm{km})$. The mean temperature for this night is the thin solid line and MSIS temperature profile is the dotted line.

close to the observed mean, except for the observed maxima at $84 \mathrm{~km}$. The mean of the five lidar profiles will also contain a residual from longer period wave motions such as tides. We then subtracted the mean from each profile to determine the wave perturbations. In Fig. 3, the temperature perturbations about the nightly mean showed downward motion of the temperature maxima and minima with time, although the rate of descent was different at different altitudes, and the variance at small scales made identifying the maxima and minima somewhat difficult. The last profile shows increased short scale perturbations in both beams, but the error is also larger, so these may be mostly noise. The RMS values of the fluctuations in each profile (after subtracting the mean) are shown in Table 1. Beam 2 showed increased wave activity at 21:06 UT at about $3 \mathrm{~km}$ vertical wavelength in temperature and especially in the vertical 

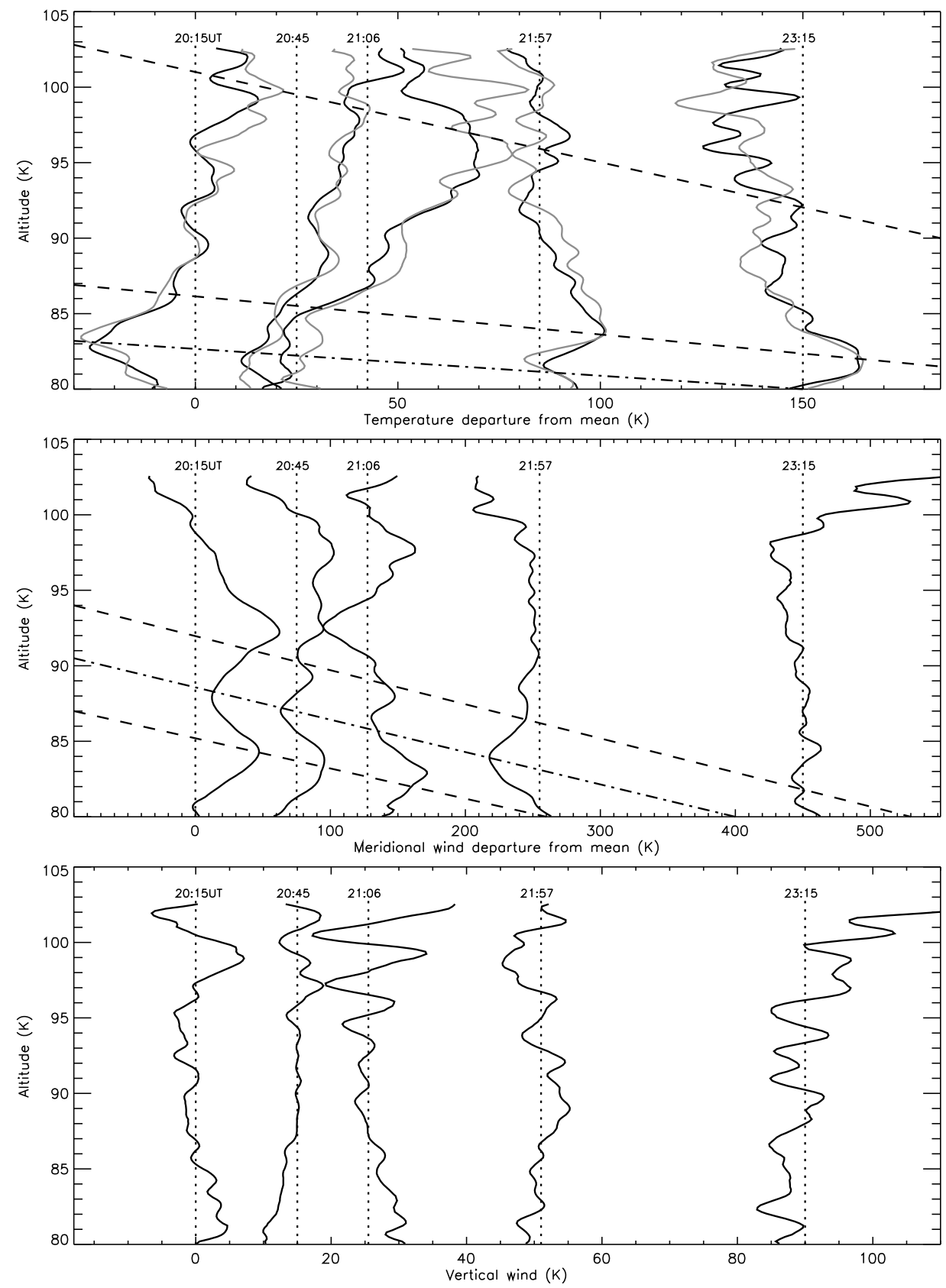

Fig. 3. Profiles of temperature (a) and meridional (b) and vertical (c) wind perturbations for both beams with the mean of the five profiles removed. Note the similarity between the two beams in temperature and the generally downward phase progression likely due to a longer-period, larger-vertical wavelength wave. We have marked possible downward moving maxima (dash line) and minima (dash-dot). In the meridional wind, the phase progression is not as clear for the larger scale perturbations even though these have large amplitudes (see Fig. 2). There does appear to be coherent shorter vertical wavelength waves during the first four profiles. There is enhanced short vertical wavelength wave activity at 23 UT in all plots and at 21 UT in beam 2 temperature and vertical wind.

wind. The meridional winds displayed significant variability but only showed clear downward phase progression for the first 2 hours below $87 \mathrm{~km}$. The time extent of the data is too short to estimate the longer period motions, especially given that the semidiurnal tide is strong and variable at this latitude (Riggin et al., 2003; Williams et al., 2006).

\section{Discussion}

We can calculate the buoyancy frequency $(\mathrm{N})$ from the temperature profile with the standard formula: $N^{2}=$ $g / T\left(d T / d z+\Gamma_{\mathrm{ad}}\right)$, where $\Gamma_{\mathrm{ad}}$ is the adiabatic lapse rate $(9.5 \mathrm{~K} / \mathrm{km}$ at $90 \mathrm{~km}), g$ is the acceleration of gravity, and
$T$ is the temperature in $\mathrm{K}$. In the simple theory, the atmosphere is susceptible to convective instability when $N^{2}<0$ and to additional instabilities at smaller wave amplitudes. The Richardson number ( $\mathrm{Ri}$ ) is defined as the square of the buoyancy frequency divided by the sum of the squares of the zonal and meridional wind gradients. Dynamic instability generally occurs when $0<\mathrm{Ri}<1 / 4$, but, as above, instabilities can also occur due to gravity waves at higher $\mathrm{Ri}$ (Fritts and Alexander, 2003). We do not have a lidar measurement of the zonal wind shear, so our calculations will overestimate $\mathrm{Ri}$ and underestimate the size of the regions of instability. Figure 4 shows where profiles of $N^{2}$ reach 0 and 


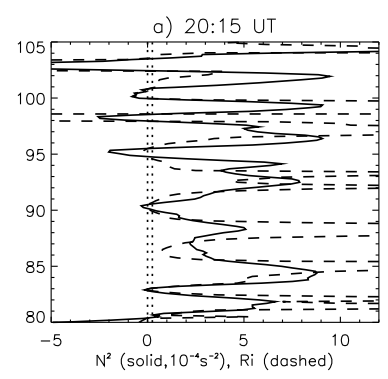

c) $21: 57$ UT

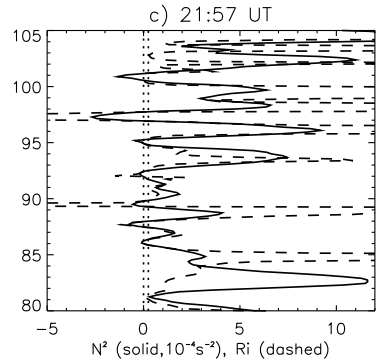

b) $20: 45$ UT

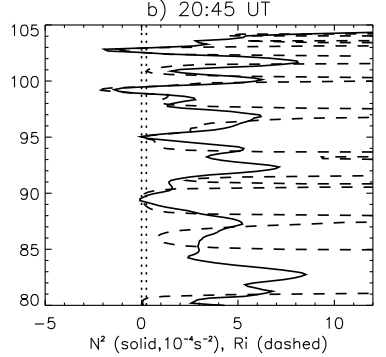

d) $23: 15$ UT

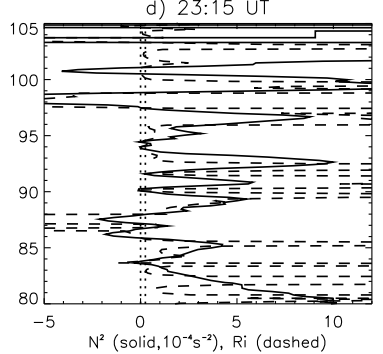

Fig. 4. Profiles of the buoyancy frequency and Richardson number for the north beam for profiles $1,2,4$, and 5 . The third profile is shown in Fig. 6. Note the multiple regions of potential instability persisting over successive measurement times at the same altitude. The two dotted lines mark where the $\mathrm{Ri}<1 / 4$ and $N^{2}<0$ and, which are the conditions for dynamic and convective instability. Convective instability likely dominates as the regions with $\mathrm{Ri}<0$ are larger than the regions with $0<\mathrm{Ri}<1 / 4$.

$\mathrm{Ri}$ is less than $1 / 4$ for the north beam. There was a region of small $N^{2}$ at $90 \mathrm{~km}$ throughout the night with the largest regions of possible instability during the second half of the night.

The last two profiles show a large negative temperature gradient extending from $84 \mathrm{~km}$ to $94 \mathrm{~km}$ in the 21:57 UT profile that moved downward $1 \mathrm{~km}$ by $23: 15 \mathrm{UT}$. The large negative temperature gradient was not quite adiabatic on its own, but perturbations by short vertical wavelength gravity waves formed multiple superadiabatic regions in this region. The presence of this unstable region does not appear to have affected the wave field significantly, as the RMS perturbations shown in Table 1 are similar for most of the profiles. This is consistent with earlier studies at ALOMAR, where a region of instability that descended with the phase front of a very-large semidiurnal tide seemed to have little effect on gravity wave propagation (Williams et al., 2006). It may be that most of the gravity waves have spatial or temporal scales that are larger than the regions of instability, so that they can propagate through relatively unaffected.

We had good quality data over an altitude range of $20 \mathrm{~km}$ from $82 \mathrm{~km}$ to $102 \mathrm{~km}$ which allowed us to determine the vertical wavelength spectrum from $2 \mathrm{~km}$ to $20 \mathrm{~km}$ for each temperature and wind profile. The vertical wavelengths derived are most robust for wavelengths between 4 and 10 $\mathrm{km}$. We used a Lomb periodogram for display convenience. There were significant perturbations at 10 to $12 \mathrm{~km}$ wavelength for both temperature and wind for most profiles except for 21:06 UT when the strongest response was at 20 $\mathrm{km}$ wavelength (not shown). The first 2 profiles had perturbations at $4.5 \mathrm{~km}$ in the temperature and vertical wind. A $7 \mathrm{~km}$ wavelength perturbation became very strong in the

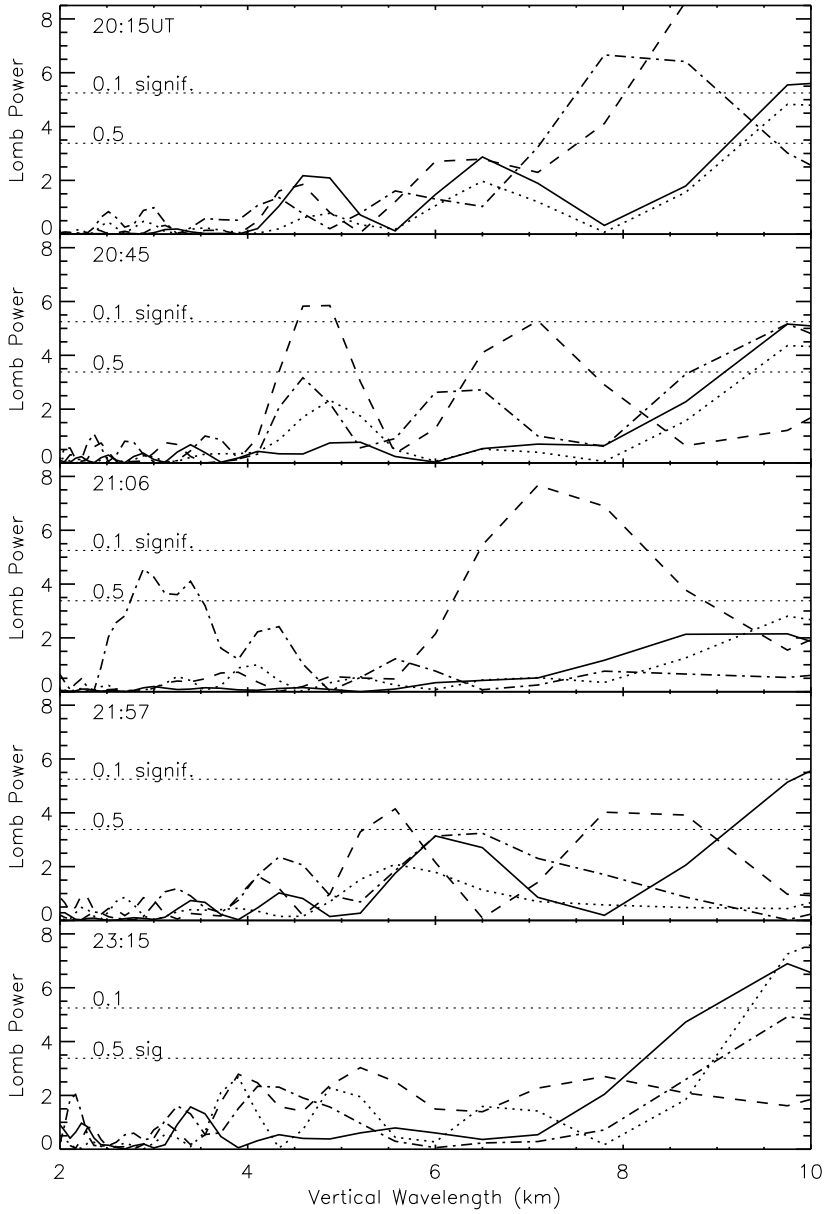

Fig. 5. Plots of Lomb power as a function of vertical wavelength for the five measurement times for temperature in beam 1 (solid line) and beam 2 (dotted), and meridional (dashed) and vertical (dash-dot) wind. The 0.5 and 0.1 significance levels are shown.

meridional wind profiles at 20:45 and 21:06. The vertical wind had a very strong $3 \mathrm{~km}$ signal at 21:12 UT that will be discussed below. This perturbation shows only moderate significance in these plots, but that is because it only existed above $90 \mathrm{~km}$, where it rapidly increases with altitude. If you do the calculation just for the altitudes where the wave was present, it is much more significant. There was also a weaker $\sim 6.5 \mathrm{~km}$ signal at 22 UT in the vertical wind.

The most unusual feature during this night was the signal in the vertical wind with a $3 \mathrm{~km}$ vertical wavelength at 21:06 UT so we will discuss this in more detail. Figure 6(a) shows all of the perturbation profiles with error bars on the vertical profile. The $3 \mathrm{~km}$ signal in the temperature profile was superimposed on a large background gradient and perturbations at larger vertical scales, so we subtracted the nightly mean profile and then applied a high pass filter to remove wavelengths greater than $4 \mathrm{~km}$. The vertical resolution is 1 $\mathrm{km}$, so the Nyquist wavelength is $2 \mathrm{~km}$, which is rather close to the $3 \mathrm{~km}$ wavelength. We repeated the calculation at 0.5 $\mathrm{km}$ resolution and obtained the same wavelength. We know of no instrumental effects that would produce an oscillatory signal in the data and the perturbation amplitude was larger than the photon noise by about a factor of two. The 


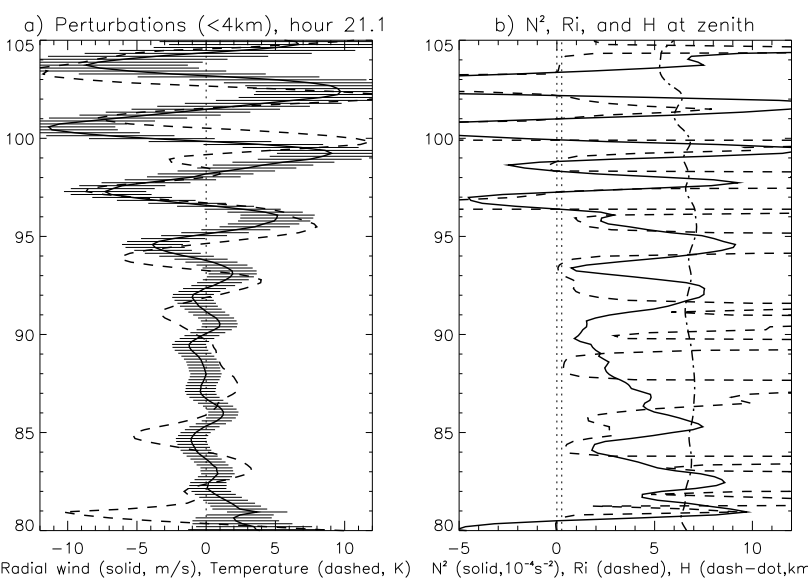

Fig. 6. a) Expanded view of the large vertical wind perturbation (solid line with error bars) at 21:00 to 21:12 UT for vertical scales less than $4 \mathrm{~km}$. The dashed line is the temperature perturbation in that beam. b) This shows the square of the buoyancy frequency (solid) and scale height (dash-dot) calculated using the temperature profile for the zenith beam. The dashed line is the Richardson number computed using the vertical beam temperature and the calculated meridional wind. The two dotted lines mark where the $\mathrm{Ri}<1 / 4$ and $N^{2}<0$ and, which are the conditions for dynamic and convective instability.

perturbation appeared in both temperature and wind even though they were calculated from different ratios of the 3 transmitted frequencies. So we believe the signal was a real atmospheric wave.

In the vertical beam, the wave grew out of the noise threshold at $90 \mathrm{~km}$ with amplitudes of no more than several $\mathrm{m} / \mathrm{s}$ or K. It appeared to grow exponentially with height to amplitudes of $10 \mathrm{~m} / \mathrm{s}$ and $10 \mathrm{~K}$ at $102 \mathrm{~km}$ altitude. The exponential growth scale was $5 \mathrm{~km}\left(e^{\mathrm{z} / 5 \mathrm{~km}}\right)$. This is much shorter than the expected undamped growth scale equal to twice the scale height $(2 \mathrm{H} \sim 12 \mathrm{~km})$. The north beam does not show this strong perturbation, so this appears to be a localized wave. One possibility is that the localized wave packet propagated obliquely into the lidar beam, resulting in the larger-than-expected growth rate.

This profile was calculated from 12 minutes of data, so the apparent wave period must have been larger than 12 min, so we will use the medium frequency wave equations. At $97 \mathrm{~km}$, the vertical wind perturbation, $w^{\prime}$, was $7 \mathrm{~m} / \mathrm{s}$, the temperature perturbation, $T^{\prime}$, was $8 \mathrm{~K}$, and the average buoyancy frequency for the profile, $N$, was $0.017 \mathrm{~s}^{-1}$. We can then estimate the zonal wind perturbation from the polarization relation for medium frequency gravity waves,

$$
\begin{aligned}
u^{\prime} \sim T^{\prime} / T_{0} g / N & =(8 \mathrm{~K}) /(220 \mathrm{~K})\left(9.54 \mathrm{~m} / \mathrm{s}^{2}\right) /\left(0.017 \mathrm{~s}^{-1}\right) \\
& =20 \mathrm{~m} / \mathrm{s}
\end{aligned}
$$

Also, $\lambda_{z}=2 \pi(c-\bar{u}) / N$, where $c$ is the horizontal phase speed and $\bar{u}$ is the mean wind. So $c-\bar{u}=$ $\left(0.017 \mathrm{~s}^{-1}\right)(3000 \mathrm{~m}) /(6.28)=8 \mathrm{~m} / \mathrm{s}$ and, hence, $u^{\prime}>c-\bar{u}$, indicating wave instability. The lapse rate is superadiabatic in multiple layers at $97 \mathrm{~km}$ and above (Fig. 6(b)) indicating instability as well. It is interesting that the wave continues to grow exponentially with height while successive wave fronts are steep enough to break. The wave is not evident in the next profile after a $30 \mathrm{~min}$ data gap, so it may be that the wave has just reached maximum amplitude and it breaks during the data gap.

The intrinsic frequency, $\omega=N w^{\prime} / u^{\prime}=0.007 \mathrm{~s}^{-1}$, which is a 15 min period. An $8 \mathrm{~K}$ temperature perturbation would correspond to a $0.84 \mathrm{~km}$ adiabatic vertical displacement. The vertical wind perturbation, $w^{\prime}$, is equal to the vertical displacement multiplied by the intrinsic frequency, $\omega$. So $\omega=\left(7 \mathrm{~ms}^{-1}\right) /(840 \mathrm{~m})=0.008 \mathrm{~s}^{-1}$, which is close to the other estimate for $\omega$. In order to resolve the wave, the observed period must have been longer than the $12 \mathrm{~min}$ averaging period. Looking at $15 \mathrm{~min}$ resolution data (not shown), the vertical wind perturbations appear to have moved downwards by $1.5 \mathrm{~km}$ in about $15 \mathrm{~min}$, giving an observed period of $30 \mathrm{~min}$ for a $3 \mathrm{~km}$ vertical wavelength wave. This is only a rough estimate due to the limited time resolution. If the observed period is greater than the intrinsic period, then the GW is most likely propagating against the local wind field.

The temperature and vertical wind perturbations are expected to be in quadrature, but the phase difference varies with height. From 90 to $97 \mathrm{~km}$, the temperature maxima are about a quarter-wavelength below the vertical wind maxima, which is the relationship expected for an upward propagating wave. At $97 \mathrm{~km}$, the vertical wind and temperature were in phase and the temperature maxima were above than the vertical wind maxima at $100 \mathrm{~km}$. The phase relationship departed from the theory in the region where there were multiple unstable layers. One possibility may have been the presence of secondary waves generated in the unstable regions that superimposed with the upward propagating wave to account for the phase variation. Recall that the temperature signal was a small perturbation on top of larger signals, so the filtering and background subtraction could account for the lack of the expected phase difference.

\section{Conclusions}

This night had the largest region of near-adiabatic lapse rates that we have yet observed with the Weber lidar in Norway with a vertical extent of $10 \mathrm{~km}$ in two profiles separated by over an hour. In Colorado in 1997, the CSU sodium lidar observed an even larger near-adiabatic region that covered about $10 \mathrm{~km}$ in altitude and lasted for 4 hours (Williams et al., 2002). The CSU lidar is able to measure such large regions of instability and they are relatively rare, but it has lacked to resolution to measure small regions of instability $(<2 \mathrm{~km})$ or waves with short periods or vertical wavelengths.

The DELTA campaign night also had some of the strongest waves with short vertical wavelengths observed to date near the mesopause at ALOMAR. Generally, the wintertime observations at ALOMAR have been dominated by longer wavelength, longer period waves such as tides (Williams et al., 2006) with shorter vertical wavelengths or periods more common in summer (Williams et al., 2004). Hansen and Hoppe (1996) observed both a significant semidiurnal tide and gravity waves with significant vertical amplitudes but long vertical wavelengths and short periods using the European incoherent scatter radar (EISCAT) radar and sodium lidar measurements in August 1989. Other measurements from EISCAT revealed significant ver- 
tical wind amplitudes for gravity waves with $\sim 30$ min period, but these had long vertical wavelengths and no systematic growth with altitude (Mitchell and Howells, 1998).

In spite of this high activity at short vertical scales, strong temperature and wind shears, and auroral activity on this night, the sodium density profiles (see Fig. 1) show no layering similar to the sporadic sodium layers observed in summer. This agrees with the current theory for sporadic metal layers that involves layers of metal ions that are concentrated by wave-induced wind shears and pushed downward to form narrow neutral metal layers (Cox and Plane, 1998). As expected, strong wave dynamics alone were insufficient to produce narrow layers, even in the presence of aurora, as on this night.

Acknowledgments. This work was supported at CSU and CoRA/ NWRA in part by the AFOSR under contract F49620-03-C-0045, by the NSF under grants ATM-0137354, ATM-0137555, and ATM-0436703, and NASA contract NAG5-02036. We acknowledge the important support of the Andoya Rocket Range and the ALOMAR observatory.

\section{References}

Cox, R. M. and J. M. C. Plane, An ion-molecule mechanism for the formation of neutral sporadic Na layers, J. Geophys. Res., 103, 63496359, 1998.

Fritts, D. C. and M. J. Alexander, Gravity wave dynamics and effects in the middle atmosphere, Rev. Geophys., 41, 1003, doi:10.1029/ 2001RG000106, 2003.

Fritts, D. C., B. P. Williams, C. Y. She, J. D. Vance, M. Rapp, F. J. Lübken, A. Müllemann, F. J. Schmidlin, and R. A. Goldberg, Observations of extreme temperature and wind gradients near the summer mesopause during the MaCWAVE/MIDAS rocket campaign, Geophys. Res. Lett., 31, LS24S06, doi:10.1029/2003GL019389, 2004.

Goldberg, R. A., D. C. Fritts, B. P. Williams, F. J. Lübken, M. Rapp, W. Singer, R. Latteck, P. Hoffmann, A. Müllemann, G. Baumgarten, F. J. Schmidlin, C. Y. She, and D. A. Krueger, The MaCWAVE/MIDAS rocket and ground-based measurements of polar summer dynamics: Overview and mean state structure, Geophys. Res. Lett., 31, LS24S02, doi:10.1029/2004GL019411, 2004.

Goldberg, R. A., D. C. Fritts, F. J. Schmidlin, B. P. Williams, C. L. Croskey, J. D. Mitchell, M. Friedrich, J. M. R. III, U. Blum, and K. H. Fricke, The MaCWAVE program to study gravity wave influences on the polar mesosphere, Ann. Geophys., 24, 1159-1173, doi:1432-0576/ag/200624-1159, 2006.

Hansen, G. and U. P. Hoppe, Investigation of the upper mesospheric dynamics under late polar summer conditions by EISCAT and LIDAR, $J$. Atmos. Terr. Phys., 58, 317-335, 1996.

Hecht, J. H., Instability layers and airglow imaging, Rev. Geophys., 42,
RG1001, doi:10.1029/2003RG000131, 2004.

Hecht, J. H., A. Z. Liu, R. L. Bishop, J. H. Clemmons, C. S. Gardner, M. F. Larsen, R. G. Roble, G. R. Swenson, and R. L. Walterscheid, An overview of observations of unstable layers during the Turbulent Oxygen Mixing Experiment (TOMEX), J. Geophys. Res., 109, D02S01, doi:10.1029/2002JD003123, 2004.

Kurihara, J., T. Abe, K. Oyama, E. Griffin, M. Kosch, A. Aruliah, K. Kauristie, Y. Ogawa, S. Komada, and N. Iwagami, Observations of the lower thermospheric neutral temperature and density in the DELTA campaign, Earth Planets Space, 58, this issue, 1123-1130, 2006.

Li, F., A. Z. Liu, and G. R. Swenson, Characteristics of instabilities in the mesopause region over Maui, Hawaii, J. Geophys. Res., 110, D09S12, doi:10.1029/2004JD005097, 2005.

Meriwether, J. W. and A. J. Gerrard, Mesosphere inversion layers and stratosphere temperature enhancements, Rev. Geophys., 42, RG3003, doi:10.1029/2003RG000133, 2004.

Mitchell, N. J. and V. S. C. Howells, Vertical velocities associated with gravity waves measured in the mesosphere and lower thermosphere with the EISCAT VHF radar, Ann. Geophys., 16, 1367-1379, 1998.

Nozawa, S., Y. Ogawa, A. Brekke, T. Tsuda, C. M. Hall, H. Miyaoka, J. Kurihara, T. Abe, and R. Fujii, EISCAT observational results during the DELTA campaign, Earth Planets Space, 58, this issue, 1183-1191, 2006.

Riggin, D. M., C. K. Meyer, D. C. Fritts, M. J. Jarvis, Y. Murayama, W. Singer, R. A. Vincent, and D. J. Murphy, MF radar observations of seasonal variability of semidiurnal motions in the mesosphere at high northern and southern latitudes, J. Atmos. Sol.-Terr. Phys., 65, 483-493, 2003.

She, C. Y., J. D. Vance, B. P. Williams, D. A. Krueger, H. Moosmüller, D. Gibson-Wilde, and D. C. Fritts, Lidar studies of atmospheric dynamics near polar mesopause, EOS, 83, 289-293, 2002.

She, C. Y., T. Li, R. L. Collins, T. Yuan, B. P. Williams, T. D. Kawahara, J. D. Vance, P. Acott, and D. A. Krueger, Tidal perturbations and variability in the mesopause region over Fort Collins, CO (41N, 105W): Continuous multi-day temperature and wind lidar observations, Geophys. Res. Lett., 31, L24111, doi:10.1029/2004GL021165, 2004.

Williams, B. P., M. A. White, D. A. Krueger, and C. Y. She, Observation of a large amplitude wave and inversion layer leading to convective instability in the mesopause region over Fort Collins, CO (41N, 105W), Geophys. Res. Lett., 29, 1850-1853, doi:10.1029/2001GL014514, 2002.

Williams, B. P., D. C. Fritts, L. Wang, C. Y. She, J. D. Vance, F. J. Schmidlin, R. A. Goldberg, A. Müllemann, and F. J. Lübken, Gravity waves in the arctic mesosphere during the MaCWAVE/ MIDAS summer rocket program, Geophy. Res. Lett., 31, L24S05, doi: 10.1029/2004GL020049, 2004.

Williams, B. P., D. C. Fritts, C. Y. She, and R. A. Goldberg, Gravity wave propagation through a large semidiurnal tide and instabilities in the mesosphere and lower thermosphere during the winter 2003 MaCWAVE rocket campaign, Ann. Geophys., 24, 1199-1208, doi:SRef-ID: 14320576/ag/2006-24-1199, 2006.

B. P. Williams (e-mail: biff@cora.nwra.com), D. C. Fritts, J. D. Vance, C.-Y. She, T. Abe, and E. Thrane 